\title{
ASCI 2010 contrast media guideline for cardiac imaging: a report of the Asian Society of Cardiovascular Imaging cardiac computed tomography and cardiac magnetic resonance imaging guideline working group
}

\author{
ASCI CCT \& CMR Guideline Working Group • \\ Masahiro Jinzaki $\cdot$ Kakuya Kitagawa $\cdot$ I-Chen Tsai • \\ Carmen Chan $\cdot$ Wei Yu $\cdot$ Hwan Seok Yong $\cdot$ Byoung Wook Choi
}

Received: 12 August 2010/Accepted: 14 August 2010/Published online: 8 October 2010

(C) The Author(s) 2010. This article is published with open access at Springerlink.com

\begin{abstract}
The use of contrast media for cardiac imaging becomes increasing as the widespread of cardiac CT and cardiac MR. A radiologist needs to carefully consider the indication and the injection protocol of contrast media to be used as well as the possibility of adverse effect. There are several guidelines for contrast media in western countries. However, these are focusing the adverse effect of contrast media. The Asian Society of Cardiovascular Imaging, the only society dedicated to cardiovascular imaging in Asia, formed a Working Group and created a guideline, which summarizes the integrated knowledge of contrast media for cardiac imaging. In cardiac imaging, coronary artery evaluation is feasible by non-contrast MR angiography, which can be an alternative examination in high risk patients for the use of iodine contrast media. Furthermore, the
\end{abstract}

M. Jinzaki ( $₫)$

Department of Diagnostic Radiology, Keio University

School of Medicine, Tokyo, Japan

e-mail: jinzaki@rad.med.keio.ac.jp

\section{K. Kitagawa}

Department of Diagnostic Radiology, Mie University

School of Medicine, Tsu, Japan

I.-C. Tsai

Department of Radiology, Taichung Veterans General

Hospital, Taichung, Taiwan

C. Chan

Division of Cardiology, Department of Medicine,

Queen Mary Hospital, Hong Kong, China body habitus of Asian patients is usually smaller than that of their western counterparts. This necessitates modifications in the injection protocol and in the formula for calculation of estimated glomerular filtration rate. This guideline provided fundamental information for the use of contrast media for Asian patients in cardiac imaging.

Keywords Contrast media - Adverse effect . Injection protocol

\section{Introduction}

As the use of contrast media in cardiac imaging is becoming more common, a radiologist needs to carefully consider the indication and the injection

W. Yu

Department of Radiology, Beijing Anzhen Hospital, Capital Medical University, Beijing, China

H. S. Yong

Department of Radiology, Korea University Guro Hospital, Seoul, Korea

B. W. Choi

Department of Radiology, Research Institute of Radiological Science, Severance Hospital, Yonsei University Health System, Seoul, Korea 
protocol of contrast media to be used as well as the possibility of adverse effect. There are several guidelines focusing the adverse effect of contrast media $[1,2]$. However, there is no guideline, which summarizes the integrated knowledge of contrast material for cardiac imaging, especially for patients of Asian origin. In cardiac imaging, coronary artery evaluation is feasible by non-contrast MR angiography, which can be an alternative examination in high risk patients for the use of iodine contrast media. Furthermore, the body habitus of Asian patients is usually smaller than that of their western counterparts. This necessitates modifications in the injection protocol and in the formula for calculation of estimated glomerular filtration rate (eGFR) [3, 4]. The major purpose of this manual is to provide fundamental information for the use of contrast media for Asian patients in cardiac imaging.

For the usage of contrast material, the basic knowledge for contrast material injection is very important to obtain optimal contrast images. The knowledge for adverse reaction is inevitable for the safe patient care. Among several adverse reactions, contrast induced nephropathy (CIN) is almost specific to iodinated contrast media, while nephrogenic systemic fibrosis (NSF) is specific to gadolinium contrast media. On the other hands, adverse reactions other than CIN and NSF is similar in both iodinated and gadolinium contrast material. Thus, this guideline consists of four chapters; (1) general rule for contrast material injection, (2) adverse reaction of iodinated and gadolinium contrast material, (3) contrast induced nephropathy and (4) nephrogenic systemic fibrosis.

\section{General rule for contrast material injection}

\subsection{Computed tomography contrast material}

Contrast enhancement in a given patient is determined by 3 factors: contrast material flow rate $(\mathrm{ml} / \mathrm{s})$, contrast material volume $(\mathrm{ml})$ and contrast material iodine concentration $(\mathrm{mg} / \mathrm{ml})$ [5]. The overall contrast volume is calculated as the injection rate multiplied by the injection duration. Warming of contrast agent prior to injection decreases viscosity and allows higher injection rates at lower injection pressures.

Accurate timing of the scan with respect to the arrival of the intravenous (IV) contrast in the target structures is necessary. Thus, the usage of either bolus tracking or a test bolus protocol is recommended.

\subsubsection{Coronary CT Angiography}

- The injection flow rate mainly determines the enhancement (fast dynamic peak). The contrast material volume also affects the enhancement $[6,7]$.

- Optimal images require high intraarterial opacification of more than 250 Hounsfield units (HU) [8]. Higher intracoronary attenuation value will improve the diagnostic accuracy of coronary artery stenosis $[9,10]$.

- High iodine concentration contrast agents are preferred to achieve greater contrast-to-noise ratios $[11,12]$.

- The injection duration should be as long as or slightly longer than the estimated scan duration. For very short scans, the injection duration should be at least $10 \mathrm{~s} \mathrm{[5].}$

- A biphasic injection protocol using dual-head pumps is preferable [13, 14]. It consists of a first injection of contrast at a rate of 3-6 $\mathrm{ml}$ (volume depends on scan length) and a second injection of approximately $20-40 \mathrm{ml}$ of saline at the same injection rate.

- A body weight tailored injection protocol is recommended to decrease the total contrast material volume in Asian patients. The use of $0.7 \mathrm{ml} / \mathrm{kg}$ of contrast material injected at a fixed duration of $10 \mathrm{~s}$ followed by $20 \mathrm{ml}$ of saline is feasible $[15,16]$.

\subsubsection{Other protocols}

- In CT angiography (CTA) protocols, the right heart typically appears washed out. In some clinical settings, it may be desirable to have some opacification of the right heart.

- For the right heart opacification, the saline flush may be replaced by a mixture of contrast and saline, or a triphasic injection protocol may be used [17-19].

- Triphasic protocols consist of an initial high flow rate contrast injection (3-6 ml/s), followed by a second injection of either a mixture of contrast 
and saline $(3-6 \mathrm{ml} / \mathrm{s})$ or a contrast injection at lower injection rate (e.g., $2 \mathrm{ml} / \mathrm{s}$ ), followed by a third injection of a smaller volume of saline.

- Regarding the protocol for the evaluation of delayed enhancement, further studies are required.

\subsection{Magnetic resonance contrast material}

Gadolinium based contrast media (GBCM) shorten $\mathrm{T} 1$ relaxation times and thus lead to higher signal intensity on T1-weighted images. Although first-pass kinetics and late distribution of GBCM are similar to those of iodinated contrast materials for $\mathrm{CT}$; there are two distinct characteristics in GBCM compared to iodinated contrast materials. First, the signal intensities are not proportional to GBCM concentration due to substantial signal loss caused by $\mathrm{T} 2$-shortening effect at high concentrations of GBCM. Therefore, the concentration of GBCM in the blood pool or myocardium cannot be calculated directly from the signal intensity on MRI [20]. Second, due to the much smaller contrast volume required for first-pass imaging, improving effect of high injection rate on bolus profile is limited for MRI compared to CT [21]. For example, when you administer a single dose of GBCM to a patient with $50 \mathrm{~kg}$ body weight (i.e. $10 \mathrm{ml}$ ), increasing injection rate from 4 to $5 \mathrm{ml} / \mathrm{s}$ shortens the injection duration only slightly (i.e. $0.5 \mathrm{~s})$.

The injection protocol for myocardial perfusion MRI is usually used as a dose of $0.05-0.1 \mathrm{mmol} / \mathrm{kg}$ with injection rate of 3-7 ml/s, followed by at least $30 \mathrm{ml}$ saline flush $(5-7 \mathrm{ml} / \mathrm{s})$. For delayed gadolinium enhancement MRI, a total dose of $0.1-0.2 \mathrm{mmol} /$ $\mathrm{kg}$ is administered.

\section{Adverse effects of iodinated and gadolinium contrast medium}

\subsection{Patient selections and preparation strategies}

Before the administration of contrast media, the referring physician and the radiologist should consider the following issues: (1) Assessment of patient risk versus potential benefit of the contrast-assisted examination. (2) Imaging alternatives that would provide the same or better diagnostic information.

(3) Prevention of adverse events.

\subsubsection{Risk factors for adverse intravenous contrast material reactions}

- The history obtained should focus on identification of factors that may indicate either a contraindication to contrast media use or an increased likelihood of a reaction.

- Severe, life-threatening reactions, although rare, can occur in the absence of any specific risk factors with any type of media [1].

- Risk factors for adverse reactions to contrast media are summarized in Table 1.

- In pregnant patients, it is unclear how iodinated or gadolinium contrast agents will affect the fetus, these agents should be administered only with extreme caution. Free iodine in radiographic contrast medium given to the mother has the potential to depress fetal/neonatal thyroid function. Neonatal thyroid function should be checked during the 1 st week if iodinated contrast media have been given during pregnancy. No effect on the fetus has been seen after gadolinium contrast media [22].

- In lactating patients, breast feeding may continue normally when iodinated or gadolinium agents are given to the mother [22].

Table 1 Risk factors for adverse intravenous contrast material reactions

\begin{tabular}{lc}
\hline Iodinated contrast media & Gadolinium contrast media \\
\hline $\begin{array}{l}\text { Patients at increased risk of reaction } \\
\text { Previous moderate or severe }\end{array}$ & $\begin{array}{l}\text { Previous moderate or severe } \\
\text { acute reaction to gadolinium }\end{array}$ \\
$\begin{array}{l}\text { acute reaction to iodine } \\
\text { contrast agent }\end{array}$ & contrast agent \\
Asthma & Asthma \\
$\begin{array}{ll}\text { Significant allergies } \\
\text { Significant cardiac disease }\end{array}$ & Significant allergies $^{\mathrm{a}}$ \\
Hyperthyroidism or other thyroid disease & \\
Multiple myeloma &
\end{tabular}

${ }^{a}$ A prior major anaphylactic response to one or more allergens

${ }^{b}$ Especially when present in those who live in iodine-deficient areas

${ }^{c}$ Especially in case of high osmolality contrast media (HOCM) administration 


\subsubsection{Preparation strategies}

- For patients at increased risk of reaction, consider an alternative test not requiring the agent. For the evaluation of coronary artery, non-contrast MR angiography can be an alternative examination [23].

- There is no concrete clinical evidence on the effectiveness of use of premedication in patients undergoing contrast enhanced CT or MRI examinations.

- However, the premedication is preferable in patients at higher risk for an acute allergic-like reaction.

- If the radiologists does intend to use premedication then a useful option can be prednisolone $30 \mathrm{mg}$ orally, given $12-2 \mathrm{~h}$ before the contrast medium [2].

- Preliminary intradermal skin testing with contrast agents is not predictive of adverse reactions, may itself be dangerous, and is not recommended $[24,25]$.

\subsection{At the time of examination}

A general category that deserves attention is emotional state. There is anecdotal evidence that severe adverse effects to contrast media or to procedures can be mitigated at least in part by reducing anxiety. It may be useful, therefore, to determine whether a patient is particularly anxious and it is important to reassure and calm that patient before contrast injection [26].

\subsubsection{To reduce the risk of adverse reactions}

- Some of the strategies to avoid contrast media induced adverse events are listed in Table 2.

\subsubsection{Extravasation}

Frequencies

- The reported incidence of IV contrast media extravasation related to power injection for CT has ranged from $0.1 \%$ to $0.9 \%(1 / 1,000$ patients to $1 / 106$ patients) [27].
Table 2 Strategies to reduce the risk of contrast medium induced adverse reactions

\begin{tabular}{ll}
\hline $\begin{array}{l}\text { Iodinated contrast } \\
\text { media }\end{array}$ & $\begin{array}{l}\text { Gadolinium contrast } \\
\text { media }\end{array}$ \\
\hline $\begin{array}{l}\text { Use a non-ionic contrast } \\
\text { medium }\end{array}$ & $\begin{array}{l}\text { Use a different gadolinium } \\
\text { contrast agent for previous } \\
\text { reactors to contrast medium }\end{array}$ \\
$\begin{array}{l}\text { Use a different iodinated agent } \\
\text { for previous reactors to } \\
\text { contrast medium }\end{array}$ & \\
\hline
\end{tabular}

- The frequency of extravasation is not related to the injection flow rate [28].

Risk factors

- Inappropriate injection site (small vessels),

- High volume of contrast media or high osmolar contrast media.

- Use of power injectors.

Type of injuries

- Most injuries are minor.

- In severe cases, ulceration, soft tissue necrosis or compartment syndrome may be observed.

- A compartment syndrome is more likely to occur after extravasation of larger volumes of contrast media [29]; however, it also has been observed after extravasation of relatively small volumes, especially when this occurs in less capacious areas (such as over the ventral or dorsal surfaces of the wrist).

To reduce the risk

- Use appropriate sized plastic cannula placed in a suitable vein to handle the flow rate.

- A test injection with normal saline.

- Use non-ionic iodinated contrast medium as far as possible.

Treatment

- Conservative management (limb elevation, use of ice packs) is adequate in most cases.

- Close clinical follow-up for several hours is essential for all patients in whom extravasations occur, since the severity and prognosis of a contrast medium extravasation injury are difficult to determine on initial evaluation of the affected site. 
- An immediate surgical consultation is indicated for any patient in whom one or more of the following signs or symptoms develops: progressive swelling or pain, altered tissue perfusion as evidenced by decreased capillary refill at any time after the extravasation has occurred, change in sensation in the affected limb, and skin ulceration or blistering.

\subsection{Type of adverse reactions}

Adverse reactions are classified into acute and delayed reactions [2]. Acute reactions are those that occur up to $1 \mathrm{~h}$ after the administration of CM. The majority of the delayed reactions occur between 1 and $72 \mathrm{~h}$ after the administration of contrast media. Subsequently occurring reactions are rare; the maximum interval is 7 days [2].

\subsubsection{Acute adverse reaction}

- The classification of severity of reactions to contrast media has been shown in Table 3 .

- The majority of adverse side effects are mild nonlife-threatening events that require only observation and supportive treatment.

- Severe adverse side effects, however, may have a mild or moderate prodrome. Nearly all lifethreatening reactions occur immediately or within the first $3 \mathrm{~h}$ after contrast media injection [30].

- Prediction of occurrence or severity is impossible, although there are some known risk factors, and anticipation and vigilance are critical [31].

- Mild reactions do not require treatment, but, as noted, they may presage or evolve into a more

Table 3 Classification of severity of reactions to contrast media

\begin{tabular}{lll}
\hline Minor & Moderate & Severe \\
\hline Nausea & Faintness & Hypotensive shock \\
Vomiting (Limited) & Vomiting (Severe) & Pulmonary edema \\
Pruritis & Urticaria (Profound) & Respiratory arrest \\
Diaphoresis & Facial edema & Cardiac arrest \\
& Laryngeal edema & Convulsions \\
& Bronchospasm & \\
\hline
\end{tabular}

severe reaction. Any patient with any reaction should, therefore, be observed for 20-30 $\mathrm{min}$, or as necessary, to ensure clinical stability and recovery.

- Moderate adverse events, by definition, are not immediately life-threatening (although they may progress to be so) but often require treatment.

- Severe adverse events are potentially or immediately life-threatening.

- Historically, adverse effects have occurred in $5-15 \%$ of all patients who receive ionic, highosmolality contrast media (HOCM) [32]. One study in Asia reported the frequency of adverse effects was $12.66 \%$ with HOCM [33].

- Use of low-osmolality ionic nonionic contrast media (LOCM) is associated with an overall incidence of adverse events of $0.2-0.7 \%$ [34, 35]. In Asia, this is reported to be $3.13 \%$ [33].

- Serious contrast reactions are rare and have occurred in 1 or 2 per $1,000(0.1-0.2 \%)$ intravascular injections of HOCM and in 1 or 2 per $10,000(0.01-0.02 \%)$ IV injections of LOCM [1]. In a report from Asia, this frequency was $0.22 \%$ of intravascular injections of ionic and $0.04 \%$ of non-ionic IV injections [33].

- The adverse event rate for gadolinium based contrast agents can range from 0.5 to $2.5 \%$ [3638].

- The management for acute adverse reactions is the same with generalized anaphylactoid reaction. This is summarized in Table 4.

- $\quad \beta$-blockers may impair the response to treatment of bronchospasm induced by contrast medium.

Table 4 Management plan for contrast medium induced acute adverse reaction

1. Call for resuscitation team

2. Suction airway as needed

3. Elevate patient's legs if hypotensive

4. Oxygen by mask (6-10 1/min)

5. Intramuscular adrenaline (1:1,000), $0.5 \mathrm{ml}(0.5 \mathrm{mg})$ in adults. Repeat as needed. In pediatric patients $0.01 \mathrm{mg} / \mathrm{kg}$ to $0.3 \mathrm{mg}$ (max. dose)

6. Intravenous fluids (e.g. normal saline, lactated Ringer's)

7. H1-blocker e.g. diphenhydramine $25-50 \mathrm{mg}$ intravenously 


\subsubsection{Delayed reactions}

- An adverse reaction which occurs $1 \mathrm{~h}$ to 1 week after contrast medium injection.

- The incidence of delayed adverse cutaneous reactions has been reported to range from 0.5 to $2 \%$ [39].

- The main types of delayed reactions are given in Table 5.

- Relatively common symptoms are nausea, vomiting, drowsiness, headache, and pruritus without urticaria, all of which are self-limited and many of which are self limiting and do not require any therapy [39].

- Skin reactions are true late adverse reactions. They are usually mild to moderate and self limiting. Delayed cutaneous reactions are not, however, associated with other acute adverse events such as bronchospasm or laryngeal edema.

- The management of late adverse reactions is identical to that of other drug induced skin reactions.

- Delayed cardiopulmonary arrest has also been reported, but this and other severe systemic reactions are probably related to etiologies other than the contrast media.

- Currently, very late reactions to gadolinium media in the form of nephrogenic systemic fibrosis (NSF) are a major concern, and are dealt with in detail in chapter $\mathrm{D}$.

\section{Contrast-induced nephropathy (iodinated contrast medum)}

Contrast medium nephrotoxicity (renal adverse reactions) is mostly associated with iodinated contrast

Table 5 Characteristics of contrast medium induced delayed adverse reactions

\begin{tabular}{|c|c|}
\hline Iodinated contrast media & Gadolinium contrast media \\
\hline \multicolumn{2}{|l|}{ Late adverse reactions } \\
\hline $\begin{array}{l}\text { Mainly skin rashes } \\
\text { Very late adverse reaction }\end{array}$ & None described \\
\hline $\begin{array}{l}\text { Thyrotoxicosis } \\
\text { (Patients with untreated Gi }\end{array}$ & $\begin{array}{l}\text { Nephrogenic systemic fibrosis } \\
\text {, disease) }\end{array}$ \\
\hline
\end{tabular}

media. The risk of nephrotoxicity is very low when gadolinium contrast media are used in approved doses.

The risk of nephrotoxicity is related to the degree of pre-existing renal disease and hydration. Clinically significant nephrotoxicity after administration of iodinated contrast media is highly unusual in patients with normal renal function. There is no standard definition for reporting contrast-induced nephrotoxicity (CIN). Definitions used have included percent change in the baseline serum creatinine (e.g., a $20-50 \%$ rise in serum creatinine) and absolute elevation from baseline $(0.5-2.0 \mathrm{mg} / \mathrm{dl})$ [40, 41].

The clinical course of CIN depends on baseline renal function, coexisting risk factors, degree of hydration, and other factors. Serum creatinine usually begins to rise within the first $24 \mathrm{~h}$ following IV contrast media administration, peaks within $96 \mathrm{~h}$ (4 days), and usually returns to baseline within 7-10 days [40]. It is unusual for patients to develop permanent renal failure, and this usually occurs in the setting of multiple risk factors.

\subsection{Risk factors for adverse intravenous contrast} medium induced nephropathy

- The major risk factors for CIN is given in Table 6.

- Serum creatinine values should be measured within 7 days of contrast media administration.

- There is no universally agreed upon threshold of serum creatinine elevation (or degree of renal

Table 6 Risk factors for contrast medium induced nephropathy
Patient related
Pre-existing renal insufficiency (serum creatinine level $>1.5 \mathrm{mg} / \mathrm{dl}$; Especially, patients with eGFR less than $30 \mathrm{ml} / \mathrm{min}$ )
Diabetes mellitus
Dehydration
Cardiovascular disease
Age over 70
Use of diuretics
Contrast medium related
High osmolality agents
Large doses of contrast medium 
dysfunction) beyond which iodinated contrast media should not be administered.

- Serum creatinine has limitations as an accurate measure of renal function because it is influenced greatly by the patient's gender, muscle mass, nutritional status, and age.

- Direct measurement of GFR with insulin or a similar clearance marker would be preferable, however, generally impractical.

- One alternative is to use a formula to calculate creatinine clearance, (estimated GFR or eGFR) based on age, gender, body weight, and serum creatinine (e.g., Cockcroft-Gault formula or Modification of Diet in Renal Disease [MDRD] formula) [42, 43].

- However, these equations are less accurate for Asians, with greater bias at estimated GFR (eGFR) less than $60 \mathrm{ml} / \mathrm{min} / 1.73 \mathrm{~m}^{2}$ [3]. This difference would be accounted for by the difference in muscle mass. African-American people probably have a greater muscle mass than Asian. Interestingly, the correction coefficients for the modification of the MDRD Study equation were considerably different even among patients of Asian origin. For example, the correction coefficient for patients of Chinese origin was 1.233 [4] and that for patients of Japanese origin 0.808 [3].

- The establishment of GFR-estimating formulae specific for patients of different race is required. For example, in Japan, new formula is recommended as follows; eGFR $\left(\mathrm{ml} / \mathrm{min} / 1.73 \mathrm{~m}^{2}\right)=$ $194 \times$ Serum creatinine ${ }^{-1.094} \times$ Age $^{-0.287} \times 0.739$ (if female) [3].

- Metformin is excreted unchanged in the urine. In the presence of renal failure, either pre-existing or induced by iodinated contrast medium, metformin may accumulate in sufficient amounts to cause lactic acidosis. Depending on serum creatinine level, metformin will have to be stopped either before or at the time of contrast medium administration.

\subsection{Prevention or amelioration}

- Consider an alternative imaging method not using iodinated contrast media. For the evaluation of coronary artery, non-contrast MR angiography can be an alternative examination [23].
- Stop nephrotoxic drugs, mannitol and loop diuretics at least $24 \mathrm{~h}$ before [44].

- Start hydration. A suitable intravenous regime is $100 \mathrm{ml} / \mathrm{h}$ of normal saline beginning $12 \mathrm{~h}$ before and continuing $12 \mathrm{~h}$ after examinations. In hot climates the volume should be increased [45].

- Stop metformin from the time of contrast medium administration or $48 \mathrm{~h}$. Only restart metformin if serum creatinine remains normal or unchanged $48 \mathrm{~h}$ after contrast medium.

- The efficacy of N-acetylcysteine (Mucomyst) or sodium bicarbonate to reduce the incidence of CIN is controversial [46-48].

\subsection{At the time of examination}

- Use low or iso-osmolar contrast media.

- Use the lowest dose of contrast medium consistent with a diagnostic result.

- Continue hydration for at least $6 \mathrm{~h}$ [49].

- In patients suffering from end-stage renal disease, there is no need for urgent dialysis [50].

- Correlation of time of the contrast medium injection with the hemodialysis session in dialysis patients is unnecessary [50].

\section{Nephrogenic systemic fibrosis (gadolinium contrast media)}

Nephrogenic systemic fibrosis (NSF) is recently reported adverse effect specific to gadolinium contrast media. Fewer cases of NSF have been reported in Asia, as compared to the US or Europe [51]. NSF is a severe, usually progressive, potentially fatal, systemic fibrotic disease, affecting the dermis, subcutaneous fasciae and striated muscles. In 2006 several groups noted a strong association between gadolinium-based contrast media (GBCM) administration and the disease [52]. In many cases, affected patients had been injected with more than one type of GBCM prior to symptoms onset. However, it must be emphasized that the frequency with which NSF has been associated with different GBCM may also have been affected if the agents were used at higher doses compared to what is recommended in their package inserts. It is advisable to use the GBCM agents within their prescribed dosages and not to overdose the 
Table 7 Risk factors for contrast medium induced Nephrogenic Systemic Fibrosis (NSF)

\begin{tabular}{ll}
\hline High risk & Low risk \\
\hline $\begin{array}{l}\text { Patients with chronic kidney } \\
\text { disease }(\mathrm{CKD}) 4 \text { and } 5\end{array}$ & $\begin{array}{c}\text { Patients with CKD 3 } \\
\left(\mathrm{eGFR}<30 \mathrm{ml} / \mathrm{min} / 1.73 \mathrm{~m}^{2}\right)\end{array}$ \\
$\begin{array}{l}\text { eGFR }<60 \mathrm{ml} / \mathrm{min} / \\
\text { Patients on dialysis }\end{array}$ \\
$\begin{array}{l}\text { Patients with acute renal } \\
\text { insufficiency in the } \\
\text { perioperative } \\
\text { liver transplantation period }\end{array}$ \\
\hline
\end{tabular}

patient. Risk factors for nephrogenic systemic fibrosis are given in Table 7 .

The etiology of NSF is still unknown but is thought to be multifactorial. The prevailing theory regarding gadolinium and NSF is that gadolinium $\left(\mathrm{Gd}^{3+}\right)$ ions are released from the Gd-chelate complex of MRI contrast agents and accumulate in tissues such as skin, thereby initiating what some have described as a "toxic" reaction. The precise pathomechanism is not yet known [53].

It is estimated that patients with eGFR $<30 \mathrm{ml} /$ $\mathrm{min} / 1.73 \mathrm{~m}^{2}$ have a $1-7 \%$ chance of developing NSF after exposure to GBCM [52, 54]. All patients should be questioned for a history of renal disease. The measurement of an eGFR within 6 weeks of the GBCM study is recommended in patients with renal disease in anyone over 60 years of age, or in patients with hypertension, diabetes mellitus.

\subsection{Preparation strategies}

- In patients with already being dialyzed.

Non-contrast MR angiography is recommended for the evaluation of coronary artery. The use of CT angiography is also possible. If a contrast-enhanced MRI examination must be performed such as for the evaluation of perfusion or delayed enhancement, avoidance of the use of those GBCM that have been most frequently associated with NSF [gadodiamide $\left(\right.$ Omniscan ${ }^{\circledR}$ ), gadopentetate dimeglumine (Magnevist $^{\circledR}$ ) and gadoversetadmide (OptiMARK $\left.{ }^{\circledR}\right]$ ) is recommended. Also, use of the lowest possible dose needed to obtain a diagnostic study is suggested. GBCM-enhanced MRI exams is recommended to be performed shortly before dialysis, as prompt post-procedural dialysis may reduce the likelihood that NSF will develop, although this has not been proved definitively to date [55].

- In patients with eGFR $<30 \mathrm{ml} / \mathrm{min} / 1.73 \mathrm{~m}^{2}$, who are not on chronic dialysis.

It is recommended that any contrast media administration be avoided if at all possible. If MRI contrast media administration is absolutely essential, judicious use of the lowest possible doses of selected GBCM (avoidance of the use of those GBCM that have been most frequently associated with NSF) is probably safest [55].

To the best of our knowledge this is the first comprehensive guide on use of contrast media amongst Asians for cardiac imaging. In summary this manual provides basic information for the use of contrast media for Asian patients in cardiac imaging. In addition to general principles of contrast material injection we have also discussed associated adverse events like contrast induced nephropathy and nephrogenic systemic fibrosis.

Open Access This article is distributed under the terms of the Creative Commons Attribution Noncommercial License which permits any noncommercial use, distribution, and reproduction in any medium, provided the original author(s) and source are credited.

\section{References}

1. American College of Radiology Manual on Contrast Media v7 http://www.acr.org/SecondaryMainMenuCategories/ quality_safety/contrast_manual.aspx

2. ESUR Guidelines on Contrast media (2006) Ver 6.0 http://www.esur.org/fileadmin/Guidelines/ESUR_2007_ Guideline_6_Kern_Ubersicht.pdf

3. Matsuo S, Imai E, Horio M et al (2009) Revised equations for estimated GFR from serum creatinine in Japan. Am J Kidney Dis 53(6):982-992

4. Ma Y, Zuo L, Chen JH et al (2006) Modified glomerular filtration rate estimating equation for Chinese patients with chronic kidney disease. J Am Soc Nephrol 17(10): 2937-2944

5. Fleischmann D, Kamaya A (2009) Optimal vascular and parenchymal contrast enhancement: the current state of the art. Radiol Clin North Am 47(1):13-26

6. Bae KT, Heiken JP, Brink JA (1998) Aortic and hepatic peak enhancement at $\mathrm{CT}$ : effect of contrast medium injection rate-pharmacokinetic analysis and experimental porcine model. Radiology 206(2):455-464

7. Cademartiri F, van der Lugt A, Luccichenti G et al (2002) Parameters affecting bolus geometry in CTA: a review. J Comput Assist Tomogr 26(4):598-607 
8. Becker CR, Hong C, Knez A et al (2003) Optimal contrast application for cardiac 4-detector-row computed tomography. Invest Radiol 38(11):690-694

9. Cademartiri F, Mollet NR, Lemos PA et al (2006) Higher intracoronary attenuation improves diagnostic accuracy in MDCT coronary angiography. AJR Am J Roentgenol 187(4):W430-W433

10. Cademartiri F, Maffei E, Palumbo AA et al (2008) Influence of intra-coronary enhancement on diagnostic accuracy with 64-slice CT coronary angiography. Eur Radiol 18(3):576-583

11. Cademartiri F, Mollet NR, van der Lugt A et al (2005) Intravenous contrast material administration at helical 16detector row CT coronary angiography: effect of iodine concentration on vascular attenuation. Radiology 236(2): 661-665

12. Rist C, Nikolaou K, Kirchin MA et al (2006) Contrast bolus optimization for cardiac 16-slice computed tomography: comparison of contrast medium formulations containing 300 and 400 milligrams of iodine per milliliter. Invest Radiol 41(5):460-467

13. Fleischmann D, Rubin GD, Bankier AA et al (2000) Improved uniformity of aortic enhancement with customized contrast medium injection protocols at CT angiography. Radiology 214(2):363-371

14. Haage P, Schmitz-Rode T, Hübner D et al (2000) Reduction of contrast material dose and artifacts by a saline flush using a double power injector in helical CT of the thorax. AJR Am J Roentgenol 174(4):1049-1053

15. Tatsugami F, Kanamoto T, Nakai G et al (2010) Reduction of the total injection volume of contrast material with a short injection duration in 64-detector row CT coronary angiography. Br J Radiol 83(985):35-39

16. Tatsugami F, Matsuki M, Inada Y et al (2010) Feasibility of low-volume injections of contrast material with a body weight-adapted iodine-dose protocol in 320-detector row coronary CT angiography. Acad Radiol 17(2):207-211

17. Mitsumori LM, Wang E, May JM et al (2010) Triphasic contrast bolus for whole-chest ECG-gated 64-MDCT of patients with nonspecific chest pain: evaluation of arterial enhancement and streak artifact. AJR Am J Roentgenol 194(3):W263-W271

18. Litmanovich D, Zamboni GA, Hauser TH et al (2008) ECG-gated chest CT angiography with 64-MDCT and triphasic IV contrast administration regimen in patients with acute non-specific chest pain. Eur Radiol 18(2):308-317

19. Lu JG, Lv B, Chen XB et al. (2009) What is the best contrast injection protocol for 64-row multi-detector cardiac computed tomography? Eur J Radiol [Epub ahead of print]

20. Lee VS (2006) Cardiovascular MR imaging: physical principles to practical protocols. In: Lee VS (ed) Tissue characteristics and contrast agents: PD, T1 and T2, 1st edn. Lippincott Williams \& Wilkins, Philadelphia, pp 42-50

21. Ishida M, Sakuma H, Murashima S et al (2009) Absolute blood contrast concentration and blood signal saturation on myocardial perfusion MRI: estimation from CT data. J Magn Reson Imag 29(1):205-210

22. Webb JA, Thomsen HS, Morcos SK (2005) Members of Contrast Media Safety Committee of European Society of Urogenital Radiology (ESUR). The use of iodinated and gadolinium contrast media during pregnancy and lactation. Eur Radiol 15(6):1234-1240

23. Sakuma H, Ichikawa Y, Chino S et al (2006) Detection of coronary artery stenosis with whole-heart coronary magnetic resonance angiography. J Am Coll Cardiol 48(10): 1946-1950

24. Yamaguchi K, Katayama H, Takashima T et al (1991) Prediction of severe adverse reactions to ionic and nonionic contrast media in Japan: evaluation of pretesting. A report from the Japanese Committee on the Safety of Contrast Media. Radiology 178(2):363-367

25. Laroche D, Aimone-Gastin I, Dubois F et al (1998) Mechanisms of severe, immediate reactions to iodinated contrast material. Radiology 209(1):183-190

26. Hopper KD, Houts PS, TenHave TR et al (1994) The effect of informed consent on the level of anxiety in patients given i.v. contrast material. AJR Am J Roentgenol 162(3): 531-535

27. Federle MP, Chang PJ, Confer S et al (1998) Frequency and effects of ionic and nonionic CT contrast media during rapid bolus injection. Radiology 206(3):637-640

28. Jacobs JE, Birnbaum BA, Langlotz CP (1998) Contrast media reactions and extravasation: relationship to intravenous injection rates. Radiology 209(2):411-416

29. Wang CL, Cohan RH, Ellis JH et al (2007) Frequency, management, and outcome of extravasation of nonionic iodinated contrast medium in 69, 657 intravenous injections. Radiology 243(1):80-87

30. Caro JJ, Trindade E, McGregor M (1991) The risks of death and of severe nonfatal reactions with high- vs lowosmolality contrast media: a meta-analysis. AJR Am J Roentgenol 156(4):825-832

31. Bush WH, Swanson DP (1991) Acute reactions to intravascular contrast media: types, risk factors, recognition, and specific treatment. AJR Am J Roentgenol 157(6):1153-1161

32. Dillman JR, Strouse PJ, Ellis JH et al (2007) Incidence and severity of acute allergic-like reactions to i.v nonionic iodinated contrast material in children. AJR Am J Roentgenol 188(6):1643-1647

33. Katayama H, Yamaguchi K, Kozuka T et al (1990) Adverse reactions to ionic and nonionic contrast media. A report from the Japanese Committee on the Safety of Contrast Media. Radiology 175(3):621-628

34. Cochran ST, Bomyea K, Sayre JW (2001) Trends in adverse events after IV administration of contrast media. AJR Am J Roentgenol 176(6):1385-1388

35. Wang CL, Cohan RH, Ellis JH et al (2008) Frequency, outcome, and appropriateness of treatment of nonionic iodinated contrast media reactions. AJR Am J Roentgenol 191(2):409-415

36. Nelson KL, Gifford LM, Lauber-Huber C et al (1995) Clinical safety of gadopentetate dimeglumine. Radiology 196(2):439-443

37. Herborn CU, Honold E, Wolf M et al (2007) Clinical safety and diagnostic value of the gadolinium chelate gadoterate meglumine (Gd-DOTA). Invest Radiol 42(1):58-62

38. Bleicher AG, Kanal E (2008) Assessment of adverse reaction rates to a newly approved MRI contrast agent: review of 23,553 administrations of gadobenate dimeglumine. Am J Roentgenol 191(6):W307-W311 
39. Christiansen C, Pichler WJ, Skotland T (2000) Delayed allergy-like reactions to X-ray contrast media: mechanistic considerations. Eur Radiol 10(12):1965-1975

40. Berkseth RO, Kjellstrand CM (1984) Radiologic contrastinduced nephropathy. Med Clin North Am 68(2):351-370

41. Cruz C, Hricak H, Samhouri F et al (1986) Contrast media for angiography: effect on renal function. Radiology 158(1): 109-112

42. Cockcroft DW, Gault MH (1976) Prediction of creatinine clearance from serum creatinine. Nephron 16(1):31-41

43. Hafez T (2007) Modification of diet in renal disease (MDRD) estimated glomerular filtration rate (eGFR) formula. Am J Cardiol 99(4):584

44. Solomon R, Werner C, Mann D et al (1994) Effects of saline, mannitol, and furosemide to prevent acute decreases in renal function induced by radiocontrast agents. N Engl J Med 331(21):1416-1420

45. Solomon RJ, Natarajan MK, Doucet S et al (2007) Cardiac angiography in renally impaired patients (CARE) study: a randomized double-blind trial of contrast-induced nephropathy in patients with chronic kidney disease. Circulation 115(25):3189-3196

46. Baker CS, Wragg A, Kumar S et al (2003) A rapid protocol for the prevention of contrast-induced renal dysfunction: the RAPPID study. J Am Coll Cardiol 41(12):2114-2118

47. Ferrario F, Barone MT, Landoni G et al (2009) Acetylcysteine and non-ionic isosmolar contrast-induced nephropathy-a randomized controlled study. Nephrol Dial Transpl 24(10):3103-3107

48. Kanter MZ (2006) Comparison of oral and i.v. acetylcysteine in the treatment of acetaminophen poisoning. Am J Health Syst Pharm 63(19):1821-1827
49. Schweiger MJ, Chambers CE, Davidson CJ et al (2007) Prevention of contrast induced nephropathy: recommendations for the high risk patient undergoing cardiovascular procedures. Catheter Cardiovasc Interv 69(1):135-140

50. Younathan CM, Kaude JV, Cook MD et al (1994) Dialysis is not indicated immediately after administration of nonionic contrast agents in patients with end-stage renal disease treated with maintenance dialysis. AJR Am J Roentgenol 163(4):969-971

51. Manufactuer's Briefing Documents for FDA AdCom Meeting Dec (2009) http://www.fda.gov/downloads/Advisory Committees/CommitteesMeetingMaterials/Drugs/Anesthetic AndLifeSupportDrugsAdvisoryCommittee/UCM183205.pdf

52. Grobner T (2006) Gadolinium-a specific trigger for the development of nephrogenic fibrosing dermopathy and nephrogenic systemic fibrosis? Nephrol Dial Transpl 21(4): 1104-1108

53. Perazella MA (2009) Current status of gadolinium toxicity in patients with kidney disease. Clin J Am Soc Nephrol 4(2):461-469

54. Marckmann P, Skov L, Rossen K et al (2006) Nephrogenic systemic fibrosis: suspected causative role of gadodiamide used for contrast-enhanced magnetic resonance imaging. J Am Soc Nephrol 17(9):2359-2362

55. Prince MR, Zhang $H$, Morris $M$ et al (2008) Incidence of nephrogenic systemic fibrosis at two large medical centers. Radiology 248(3):807-816 